\title{
Atheroma of the Larger Arteries of the Brain in a Polish Population: A Study of 600 Cases
}

\author{
M. J. MOSSAKOWSKI, Z. KRAŚNICKA AND L. IWANOWSKI
}

The Institute of Neuropathology of the Polish Academ!

of Sciences, Warsaw' (Poland)

\section{INTRODUCTION}

In 1959 the World Federation of Neurology initiated a series of epidemiological studies in cerebro-vascular disease, with the purpose of establishing the importance of various factors, including geographic, climatic, racial, nutritional and occupational. upon the spread of degenerative arterial disease of the brain, as well as on its course, frequency, intensity and distribution. Several papers on the subject have already been published (BAKER AND lANNONE 1959a-c, 1961: BAKER et al. 1960, 1961). These studies were carried out on material from two different centres, namely the United States and Norway. In Poland. epidemiological studies on brain atheroma, carried out according to WFN standards, were started in 1960. They were made upon casematerial from Warsaw, a large administrative, cultural and partly industrial centre. Studies going on at present are being carried out in two other regions of Poland - in the neighbourhood of Lublin, a rural part of the country, and in Bydgoszcz, a large industrial centre. The material, made up of data from these three centres, should be representative of Poland. Data previously collected have been published in several papers in which more detailed questions were discussed (MAJDECKI AND ZELMAN 1961: Dambska, Krasnicka, Mandybur, Wiśniewski, in the press).

The aim of the present work is to analyze the frequency and intensity of atheromatous changes in the larger arteries of the brain in the Polish population along the lines of the work done by BAKER AND IANNONE (1959a-c) and by BAKER et al. (1960) on Norwegian and American material.

\section{MATERIAL AND METHOD}

The studies were carried out on 600 unselected patients who had died in the State University Hospital No. 1 in Warsaw. As a postmortem examination is performed as a routine on almost all cases in state hospitals, our material can be considered as being representative of the average population in this region of Poland. In our material, the first decades of life are represented by only a few cases, because there is a separate Pediatric University Hospital with its own autopsy service. 
The pathological studies were carried out according to the standards laid down by the WORLD Federation of NeURology (1959). The atheromatous changes in the larger arteries of the brain were coded in the following manner, ranging:

(1) An opacity to a very thin plaque involving only a small sector of the circumference of a vessel: no narrowing of the lumen;

(2) A thin plaque involving more than half the circumference of the vessel with minimal narrowing of the lumen; or a small thick plaque causing less than $25 \%$ narrowing of the lumen;

(3) A thick plaque involving the entire circumference with narrowing of the lumen; or a localized plaque causing $25-50 \%$ narrowing;

(4) A very thick plaque involving the entire circumference with moderate or marked narrowing of the lumen (pipestem); or a localized plaque, producing more than $50 \%$ narrowing of the lumen.

Following the practice of BAKER et al. (1960) we divided all our cases into several groups, depending on the degree of advancement of the atheromatous changes. Group ' $\mathrm{O}$ ' included cases with no atheromatous changes. The sum of the changes in each case, classified according to the WFN coding system, was the basis for grouping cases with atheromatous changes. Those in which the general 'sum of atheromatosis' was less than 10 were classified as mild cases; those with 'a sum of atheromatosis' between 11 and 20 as moderate; and those with more than 21 as severe. We accepted this scheme of classification despite certain reservations as to the method because of the mechanics and subjectivity of evaluation. The principal reservation lay in the fact that the limits of the groups with severe atheromatosis were too wide, and might include cases from 21 to 112 points. It was true that none of our cases reached the maximal number of points, but it still seemed to us that there was a great difference, from both the clinical and morphological points of view, between cases with 21 and those scoring 70 points: the latter were not rare in our material. In order to avoid this inaccuracy, MAJDECKI AND ZELMAN (1961) introduced an additional group to include cases in which the sum of 'atheromatous points' was higher than 40 , and they called this, the group with the most severe atheromatosis. However, in order to make possible the comparison of our material with data obtained from American and Norwegian populations, we have retained BAKER's criteria of classification.

The series was made up of almost the same number of males and females ( 303 women and 297 men). The ages of patients varied from $11-99$ years, but the cases in the sixth, seventh and eighth decades of life were more numerous. Under 20 and over 90 years of age, there were only single cases.

\section{RESUL.TS}

Atheromatous changes were present in $68.7 \%$ of all cases. Mild atheroma was present in $27.3 \%$, moderate in $11.5 \%$, and severe in $29.8 \% ; 31.3 \%$ were devoid of any atheromatous changes. Table 1 and Fig. I show the frequency of atheromatous changes in the various age groups. Cases with the earliest atheromatous changes in the large brain arteries were observed in the third decade of life. In these, the degenerative changes in the arterial walls were of mild degree. In two cases the atheroma 


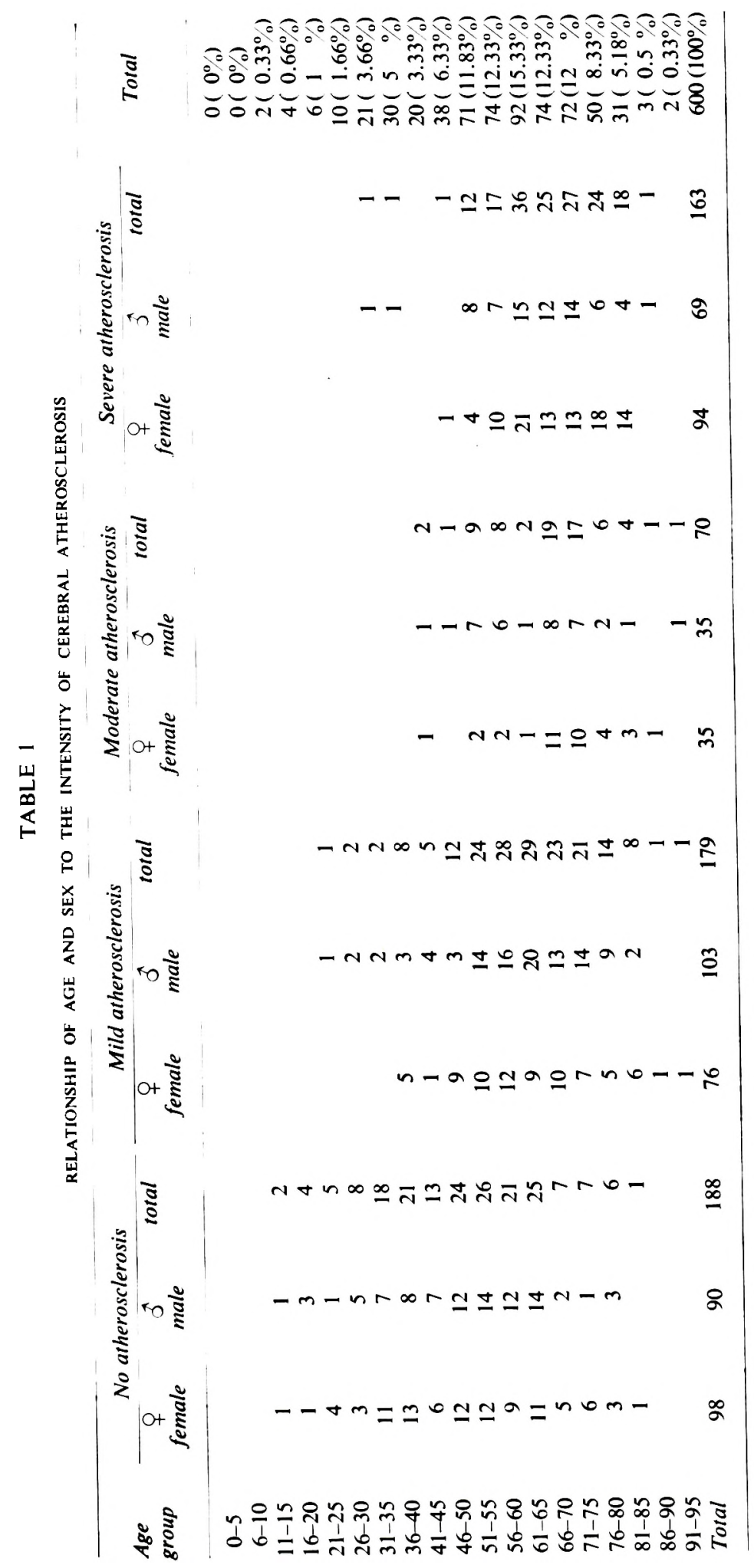

J. neurol. Sci. (1964) 1: 13-23 


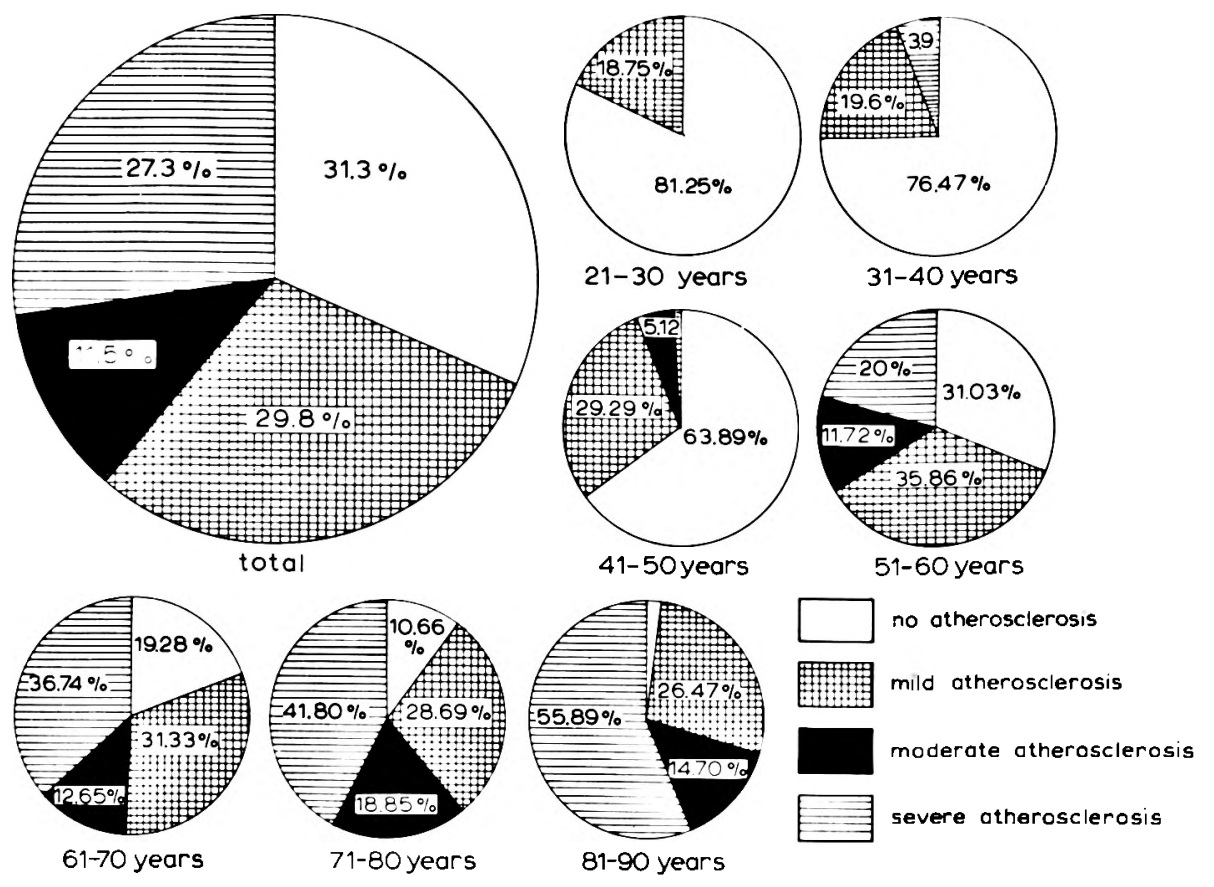

Fig. 1. Frequency of cerebral atherosclerosis in the various age-groups.

occurred in male patients with subacute bacterial endocarditis, aged respectively 23 and 30 years. The third in this group was a 29 -year-old male, who died of cerebral hemorrhage of unknown causation.

Severe atheroma in our first cases occurred in the fourth decade of life. In one this was noted in a 35-year-old male with a long history of chronic glomerulonephritis. and in the second in a 39-year-old male with a 10 -year history of arterial hypertension complicated by myocardial infarction and apoplexy.

Although atheromatous changes were noted in cases as early as in the fourth and fifth decades of life, these were in the great minority as compared with cases with no atheromatosis. In the group of patients in their sixties, atheromatous changes were found in more than two-thirds. At the same time there was an evident shift of the spectrum of atheromatous change from mild to severe grades. In the ninth decade only $2.94 \%$ of cases were free from degenerative arterial disease, and more than $55 \%$ were in the group with severe atheroma. In two cases in the tenth decade the presence of atheromatous changes was clear, but in both of these the changes were of moderate degree. Fig. 1 shows that the number of cases with no atheroma decreased progressively with age, while the number of cases with severe atheroma steadily increased.

It seemed of interest to study the frequency and severity of atheromatous changes in each of the larger cerebral arteries. Table 2 shows the frequency and degree of the atheroma within the wall of each individual component of the circle of Willis. This indicates that the internal carotids, both proximal to and at the point of trifurcation, are the most often involved by atheroma. The next commonest site is the proximal 
TABLE 2

FREQUENCY AND INTENSITY OF ATHEROMATOUS CHANGES IN LARGER CEREBRAL ARTERIES

\begin{tabular}{|c|c|c|c|c|c|c|}
\hline & Cerebral arteries & Grade I & Grade 2 & Grade 3 & Grade 4 & Total \\
\hline 1. & A. commun. anter. & 59 & 22 & 4 & - & 85 \\
\hline 2. & A. basil. (p. ant.) & 133 & 102 & 37 & 4 & 276 \\
\hline 3. & A. basil. (p. media) & 112 & 74 & 38 & 4 & 228 \\
\hline 4. & A. basil. (p. post.) & 118 & 86 & 50 & 3 & 257 \\
\hline 5. & A. cerebri ant. dext. (p. prox.) & 116 & 41 & 13 & - & 170 \\
\hline 6. & A. cerebri ant. sin. (p. prox.) & 120 & 37 & 12 & - & 169 \\
\hline 7. & A. cerebri ant. dext. (p. dist.) & 85 & 32 & 5 & - & 122 \\
\hline 8. & A. cerebri ant. $\sin$. (p. dist.) & 83 & 39 & 3 & - & 125 \\
\hline 9. & A. car. int. dext. (usque ad trifurc.) & 124 & 126 & 48 & 4 & 302 \\
\hline 10. & A. car. int. sin. (usque ad trifurc.) & 126 & 125 & 48 & 4 & 303 \\
\hline 11. & A. car. int. dext. (in trifurc.) & 134 & 131 & 50 & 3 & 318 \\
\hline 12. & A. car. int. sin. (in trifurc.) & 134 & 127 & 52 & 3 & 316 \\
\hline 13. & A. cerebri med. dext. (p. prox.) & 139 & 108 & 32 & 2 & 281 \\
\hline 14. & A. cerebri med. sin. (p. prox.) & 131 & 109 & 36 & 3 & 279 \\
\hline 15. & A. cerebri med. dext. (p. dist.) & 140 & 72 & 15 & 1 & 228 \\
\hline 16. & A. cerebri med. sin. (p. dist.) & 142 & 76 & 11 & 1 & 230 \\
\hline 17. & A. commun. post. dext. & 70 & 15 & 3 & 1 & 89 \\
\hline 18. & A. commun. post. sin. & 79 & 19 & 2 & 1 & 101 \\
\hline 19. & A. cerebri post. dext. (p. prox.) & 121 & 72 & 13 & 1 & 207 \\
\hline 20. & A. cerebri post. sin. (p. prox.) & 125 & 69 & 14 & - & 208 \\
\hline 21. & A. cerebri post. dext. (p. dist.) & 125 & 53 & 16 & - & 194 \\
\hline 22. & A. cerebri post. sin. (p. dist). & 114 & 47 & 3 & - & 164 \\
\hline 23. & A. cerebelli sup. dext. & 84 & 24 & 2 & - & 110 \\
\hline 24. & A. cerebelli sup. sin. & 69 & 24 & 2 & - & 95 \\
\hline 25. & A. vertebr. dext. & 126 & 70 & 30 & 1 & 227 \\
\hline 26. & A. vertebr. sin. & 125 & 69 & 30 & 3 & 227 \\
\hline 27. & A. cerebelli infer. post. dext. & 69 & 19 & 5 & - & 93 \\
\hline 28. & A. cerebelli infer. post. sin. & 63 & 20 & 4 & 1 & 88 \\
\hline
\end{tabular}

portion of the middle cerebral arteries, and the anterior and posterior portions of the basilar artery. The anterior communicating, the posterior communicating, and the various cerebellar arteries are the ones least often involved. As a rule, the symmetrical homonymous arteries are affected with the same frequency. The posterior communicating arteries and the superior cerebellar arteries are the only exceptions, the left posterior communicating artery and the right superior cerebellar artery being more commonly involved. Fig. 2 illustrates the severity of atheroma in the various large cerebral arteries. The degree of severity was expressed by multiplying the frequency of involvement by the severity of involvement (in pluses according to the WFN coding system). In comparing the data shown in Table 2 and Fig. 2, one sees that the degree of intensity of atheroma in our material is identical with the frequency of involvement. The arteries most often involved are at the same time the ones which are most severely affected. Thus the most advanced atheroma is found in the internal carotid arteries; in the proximal portion of the middle cerebral arteries; and in the basilar artery (posterior and anterior portions). The least advanced degree of atheroma is seen in the anterior and posterior communicating arteries, and in both cerebellar arteries.

We tried to determine in which of the arteries, atheromatous changes appeared 


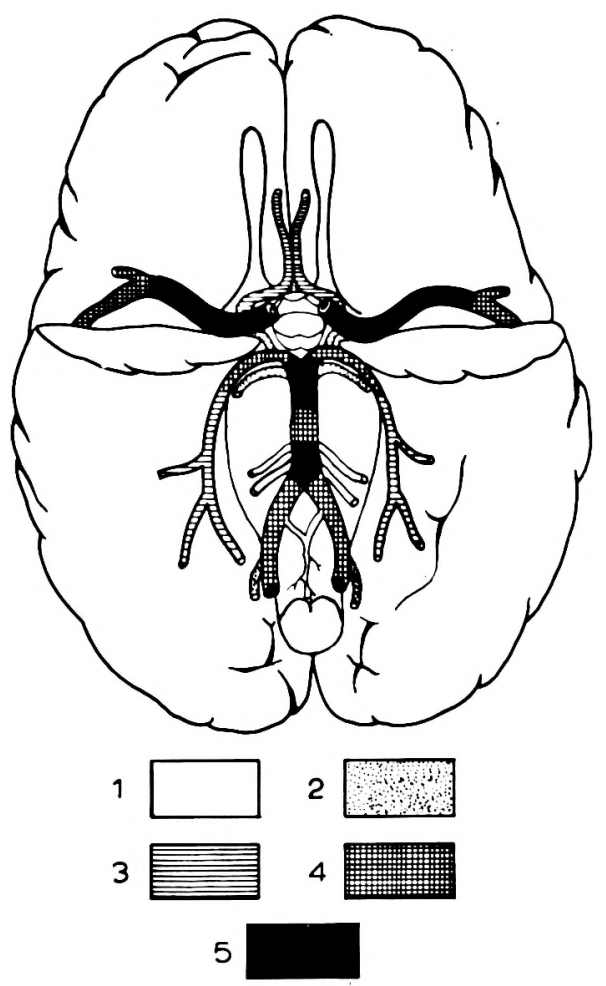

Fig. 2. Distribution of the atheromatous changes in the larger arteries of the circle of Willis. 1 : arteries not examined; 2: slight atherosclerosis; 3: moderate atherosclerosis; 4: severe atherosclerosis; 5: the most severe atherosclerosis.

TABLE 3

FREQUENCY OF CEREBRAL ATHEROSCLEROSIS ACCORDING TO THE VARIOUS AGE-GROUPS

\begin{tabular}{lrrrrrr}
\hline \multicolumn{1}{c}{ Artery } & \multicolumn{7}{c}{ Age groups* $(\%)$} \\
\cline { 3 - 7 } & $31-40$ & $41-50$ & $51-60$ & $61-70$ & $71-80$ & $81-90$ \\
\hline Anterior commun. & 1.9 & - & 11.0 & 13.2 & 27.9 & 38.2 \\
Anterior basilar & 11.8 & 10.34 & 40.7 & 60.2 & 63.9 & 79.4 \\
Middle basilar & 9.8 & 8.6 & 33.8 & 47.0 & 52.5 & 73.5 \\
Posterior basilar & 11.8 & 12.1 & 37.9 & 51.9 & 61.5 & 64.7 \\
Anterior cerebral prox. & 2.9 & 5.2 & 21.4 & 36.4 & 43.4 & 52.9 \\
Anterior cerebral dist. & 1.9 & 1.7 & 16.2 & 27.1 & 32.4 & 38.2 \\
Int. carotid before trifurc. & 10.8 & 17.2 & 44.1 & 64.7 & 72.9 & 77.9 \\
Int. carotid at trifurc. & 11.8 & 14.7 & 44.8 & 67.2 & 75.8 & 86.7 \\
Middle cerebral prox. & 7.8 & 9.4 & 40.7 & 55.4 & 72.2 & 82.4 \\
Middle cerebral dist. & 8.8 & 8.6 & 30.7 & 40.1 & 65.2 & 73.5 \\
Posterior communic. & 1.9 & 2.6 & 5.5 & 10.2 & 4.9 & 5.9 \\
Posterior cerebral prox. & 4.9 & 3.4 & 27.6 & 47.3 & 50.4 & 72.0 \\
Posterior cerebral dist. & 3.9 & 5.2 & 19.7 & 35.8 & 41.8 & 61.8 \\
Superior cerebellar & 3.9 & 1.7 & 14.1 & 21.1 & 25.0 & 22.1 \\
Vertebral & 6.9 & 10.3 & 29.0 & 48.8 & 54.5 & 61.8 \\
Posterior inf. cerebell. & 4.9 & 1.7 & 13.4 & 18.7 & 21.3 & 29.4
\end{tabular}

* Age-groups between 21-30 and 91-100 are excluded because of the very small number of cases in each of those. 
first. The slight changes observed in single cases in the third decade of life were to be seen first in the internal carotid arteries at the site of trifurcation, in the proximal portion of the middle cerebral arteries, and in the middle third of the basilar artery. Table 3 shows in what percentage of cases the individual cerebral arteries are involved according to the various age groups. This table indicates clearly that the highest percentage of atheroma in the youngest group considered (i.e. the fourth decade), occurs subsequently in the site of trifurcation of the internal carotid arteries, in the anterior and posterior portion of the basilar artery, the pre-trifurcational portion of the internal carotid, and the middle third of the basilar artery.

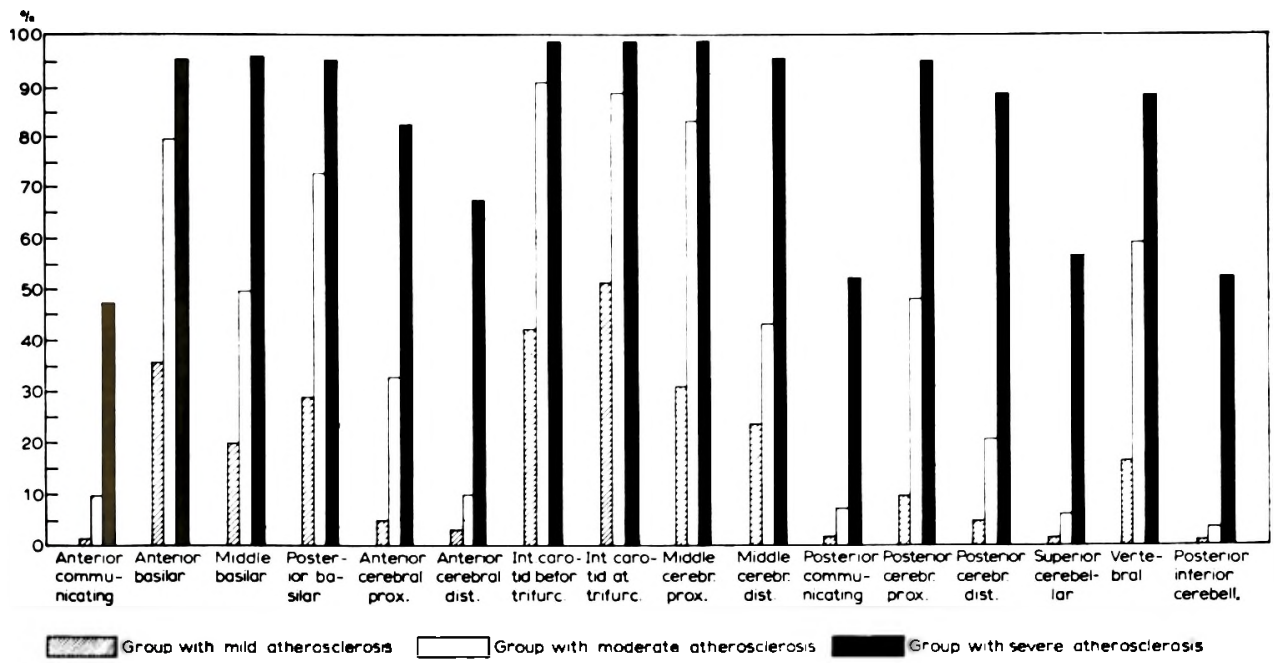

Fig. 3. Relative frequency of atheromatous changes in the larger arteries of the circle of Willis in various groups of cerebral atherosclerosis.

The next question studied was how often atheromatous changes occurred in the individual brain arteries of various groups of atheroma. In the group with severe atheroma all the larger cerebral arteries were affected in over $50 \%$ of cases. In the group with mild atheroma only, internal carotid arteries at the site of trifurcation were involved in $50^{\circ}$. Numerous large cerebral arteries in this group were involved in single cases only. Anterior and posterior communicating arteries and the cerebellar arteries were involved least frequently. This indicates that in cases with severe atheroma the pathological process was more diffuse and generalized, involving all cerebral arteries; while in the group with mild atheroma the lesions were circumscribed and limited to the large arteries which were involved most often, most severely and earliest.

Table 1 presents the data concerning the frequency and severity of atheromatous changes in various age groups, and also takes into account the sex factor. The table shows some quantitative differences in both sexes. particularly in the groups with mild and severe atheroma. There is a preponderance of men in the first group, while women make up the majority in the second. 
DISCUSSION

It is of interest to compare our results obtained with Polish subjects with the observations made by BAKER AND IANNONE $(1959 \mathrm{a}-\mathrm{c})$ in a Minnesota population and also with those of BAKER, REFSUM AND DAHL (1960). The opportunity for comparative studies was specially good as almost the same number of cases were involved, and an identical coding method was used. However, an important difference lay in the fact that our series did not contain examples from the first two decades of life which were included in the American and Norwegian studies. In general, our results are very similar to those obtained by BAKER et al. (1960). The total number of cases with atheromatous changes in the larger cerebral arteries was markedly higher in our series $(68.7 \%)$ than in the American and Norwegian researches $(54.2 \%)$, slightly higher than this in BAKER'S AND IANNONE's series $(1959 \mathrm{a}-\mathrm{c})(60 \%)$ and lower than in those described by BAKER, REFSUM AND DAHL (1960) (72.33\%). Taking into account the childhood and juvenile groups in the series of BAKER et al. as well as all of the material (both American and Norwegian), one would expect that the proportions would be less when Polish and combined American and Norwegian subjects are compared, and greater between Polish and Norwegian populations.

Quantitative comparisons of the various atheroma groups were possible only with the Norwegian material. The percentage of cases with mild $(26.5 \%)$ and severe $(28.5 \%)$ atheroma was, in BAKER's material, slightly lower than in ours. The only obvious difference found was in the group with moderate atheroma, which in our material was present in $11.5 \%$ and in BAKER's in $17.3 \%$.

The first mild atheromatous changes in our subjects occurred one and a half decades earlier than in the Norwegian group and one decade later than in the Minnesota series. The first cases with severe atheroma were noted by us in the same decade as in BAKER's material from Minnesota, but half a decade earlier than in those from Norway.

With increasing age, the frequency and severity of atheromatous changes showed only slight differences between the Polish and Norwegian populations. In the seventh decade of life the number of cases with severe atheroma was $36.74 \%$ in the Polish group and only $27.1 \%$ in the Norwegian, but the number of cases with moderate atheroma in Norway was $10 \%$, higher than in Poland. The number of cases with mild atheroma in that age-group was almost identical in both populations. In the eighth decade the differences discussed above in groups with moderate and severe atheroma were decreasing but, on the other hand, a minimal difference with slight preponderance of Norwegians appeared in the group with mild atheroma. The number of cases with no atheroma in this age group was less in BAKER's material than in ours. In the ninth decade the number of cases without atheroma was only insignificantly higher in both populations, namely 4 and $2 \%$. A much higher percentage with no atheroma in the group over 80 years was found in both the Norwegian $(12 \%)$ and the American ( $7 \%$ ) material (BAKER, IANNONE AND KINNARD 1960).

As far as the distribution of atheromatous changes in the larger brain arteries is concerned, our data are very similar to those of BAKER, REFSUM AND DAHL (1960) among Norwegians. The principal difference is in the lesser involvement of proximal 
portions of the posterior cerebral arteries and in the greater symmetry of atheromatous changes in our cases. The differences in the intensity and frequency of atheroma in the symmetrical homonymous arteries were, in our series, so slight that in no case were homonymous arteries classified among different atheromatous groups. When our data concerning the distribution of the degenerative lesions in the larger cerebral arteries are compared with those of BAKER et al. (1959a-c), one finds more severe involvement of the internal carotid arteries (at the site of bifurcation), and less damage of the proximal portions of the posterior cerebral arteries in our material than in both the American and Norwegian groups. However, the severity of atheromatous involvement of the larger cerebral arteries was much greater in our material than in that from Minnesota.

When the intensity of atheromatous changes within individual brain arteries is compared (expressed in plus signs according to the WFN coding system) the data from our material and BAKER's were very similar except that there were but few cases rated as 4 pluses in our cases. This might mean that atheromatous changes leading to marked narrowing of the calibre of larger arteries of the brain, were rarer in the Polish than in the Norwegian population.

Like Baker, IANNONE AND KINNARD (1960) in their studies on both American and Norwegian populations, we found no significant differences in frequency and intensity of atheroma as regards sex. Quantitative differences in the number of male and female cases in groups with mild and severe atheromatosis can be explained by the differing numbers of males and females in the age-groups concerned, especially the preponderance of women among the older age-groups; for instance, in the group of 170 patients over 70 years of age, 103 were women.

In our study of the time of the first appearance of atheromatous lesions, we were able to establish that the earliest changes also occurred in those arteries which were found to be involved most often and most severely. In cases in which the atheroma was less wide-spread and less advanced, irrespective of the patient's age, atheromatous changes were limited to the internal carotid, middle cerebral and basilar (anterior and posterior portion) arteries. When atheroma was intense and wide-spread, the atheromatous changes also involved other portions of the arterial tree at the base of the brain. The dependency on age of distribution of atheromatous changes seemed to be secondary. It existed inasmuch as there was an unquestionable age-factor in the generalization and intensity of atheroma.

Our observations agree with the conclusions of BAKER et al. (1959a-c, 1960, 1961) as to the relationship between the size of the vessels and the frequency and severity of atheroma. However, it seemed to us that the problem was not the size of the artery but other factors, perhaps, closely related to the blood vessel's calibre. In this connexion we had in mind the nutrition of the blood-vessel wall, (RAMSEY 1936/37), as well as the metabolism and biochemistry of the arterial tissue (KIRK 1951). We were able to establish some measure of predilection in the distribution of atheromatous changes for the sites of arterial branching (ADAMS AND VAN DER EECKEN 1953), thusconfirming a well-known view on the role of haemodynamic trauma in the pathogenesis of atheromatous degeneration. 
SUMMARY

(1) Studies were carried out upon a series of 600 unselected patients between the agelimits of 11-99 who died in the first State University Hospital in Warsaw.

(2) In $31.3 \%$ of cases there were no atheromatous changes in the larger cerebral arteries; in the remaining $68.7 \%$ of the subjects examined there were degenerative vascular changes which were classified as mild atheroma in $29.8 \%$, in $11.5 \%$ as moderate, and in $27.3 \%$ as severe.

(3) Atheromatous changes were first found in cases in the third decade of life. After that age the number of cases with brain atheroma was found to increase gradually according to the patient's age. At the same time there was a definite shift within the spectrum of atheroma from changes classified as mild to those of severe degree.

(4) The internal carotid arteries, the anterior and posterior portions of the basilar artery, and the middle cerebral arteries were the ones most severely involved. The degenerative atheromatous changes occurred in these situations most often and most early. In cases with mild atheroma there was clear-cut selectivity as to localization of a theromatous changes for the above-mentioned arteries; as the intensity of athero$\mathrm{ma}$ increased, the degenerative changes were also seen to occur in other regions of the cerebral arterial tree.

(5) There was no great difference in the frequency and intensity of atheromatous changes in the brain arteries, with regard to sex.

(6) A comparison was made with the results obtained and the data presented by BAKER et al. in their American and Norwegian series.

\section{REFERENCES}

Adams, R. D. and H. M. Van der Eecken, (1953) Vascular diseases of the brain, Ann. Rev. Med.. 4:213.

Baker A. B. and A. Iannone, (1959a) Cerebro-vascular disease, Part 1 (The large arteries of the circle of Willis), Neurology (Minneap.), 9: 321.

Baker. A. B. AND A. I ANNONE, (1959b) Cerebro-vascular disease, Part 2 (The smaller intracerebral arteries), Neurology (Minneap.), 9: 391.

BAKER, A. B. AND A. IANNONE, (1959c) Cerebro-vascular disease, Part 3 (The intracerebral arterioles), Neurolog! (Minneap.). 9: 441.

BaKer. A. B. AND A. IANNONe, (1961) Cerebro-vascular disease, Part 7 (A study of etiological mechanisms), Neurology' (Minneap.), $11: 23$.

Baker, A. B. A. Iannone and J. Kinnard, (1960) Cerebro-vascular disease, Part 5 (A comparative study of an American and a Norwegian population), World Neurology, 1: 127.

Baker, A. B., A. I ANnone and J. Kinnard, (1961) Cerebro-vascular disease, Part 6 (Relationship to disease of the heart and the aorta), Neurology (Minneap.), $11: 63$.

Baker, A. B., S. Refsum and E. Dahl, (1960) Cerebro-vascular disease, Part 4 (A study of a Norwegian population), Neurology (Minneap.), 10: 525.

DAMBSKA. M., (1963) Altération artériosclérotique cérébrale et artériosclérose des artères basales du cerveau, Acta neuropath. (Berl.), 2: 407.

KIRK, J. E., (1951) Metabolism of arterial tissue (In Proceedings of the Symposium: Studies on Atherosclerosis), J. Geront., 6: 167.

KRAŚNICKA, Z., (1963) On the clinico-pathological correlation in various states of cerebral atheromatosis (in Polish), Neuropat. pol., in the press. 
Majdecki, T., (1963) Atheromatosis of the large arteries of the brain basis and atheromatous changes on aorta and coronary arteries (in Polish), Neuropat. pol., in the press.

Majdecki, T. AND I. Zelman, (1961) Atheromatosis of the large arteries of the brain basis and atheromatous changes on aorta and coronary arteries, Part 1 (in Polish), Pol. med. Weekly, 16:1151.

MANDYBuR, T., (1963) The atheromatous changes on the intracerebral arteries, Neuropat. pol., in the press.

RAMSEY, E. M., (1936/37) Studies on the pathology of vascular disease, nutrition of the blood vessel wall, Yale, J. Biol. Med., 9: 14, 36.

WIŚNIEWSKI, W., A comparative study of the cerebral and renal arteries in cases of atheromatosis with and without hypertension, with respect to parenchymatous changes in the kidney, Neuropat. pol., in the press.

World Federation of Neurology, (1959) a Collaborative Study of Epidemiological Factors in Cerebro-Vascular Disease. Coding Guide, Antwerp. 